\title{
研究通讯
}

oreseseseses

\section{二维左对称代数的分类 *}

定义 1 在域 $K$ 上的一个线性空间 $A$, 定义一个双线性的乘法:

$$
(x, y) \longrightarrow x y, \forall x, y \in A,
$$

使得

$$
\begin{gathered}
(x y) z-x(y z)=(y x) z-y(x z), \\
\forall x, y, z \in A .
\end{gathered}
$$

则称 $A$ 是一个左对称代数.

本文用 $<, \mid,>$ 表示由<, >中的元素生成的 左对称代数, 并在 $\mid$ 后给出了具体的乘法表.

定理 1 (分类定理) 设 $A$ 是特征为零 的代数闭域上的二维左对称代数, 则 $A$ 一定 同构于下面的(互不同构)左对称代数:

i) $A$ 是可换的 (此时一定是结合代数)：

$$
\begin{aligned}
(A \text { I }) A & =\left\langle e_{1}, e_{2}\right| e_{i} e_{j} \\
& \left.=\delta_{i j} e_{i}, i, j=1,2\right\rangle ; \\
(A \text { II }) A & =\left\langle e_{1}, e_{2}\right| e_{1} e_{1}=e_{1}, e_{1} e_{2} \\
& \left.=e_{2} e_{1}=e_{2}, e_{2} e_{2}=0\right\rangle ; \\
(A \text { III }) A & =\left\langle e_{1}, e_{2}\right| e_{1} e_{1}=e_{1}, e_{1} e_{2} \\
& \left.=e_{2} e_{1}=e_{2} e_{2}=0\right\rangle ; \\
(A \text { IV }) A & =\left\langle e_{1}, e_{2}\right| e_{1} e_{1}=e_{1} e_{2}=e_{2} e_{1} \\
& \left.=e_{2} e_{2}=0\right\rangle ; \\
(A V) A & =\left\langle e_{1}, e_{2}\right| e_{1} e_{1}=e_{2}, e_{1} e_{2} \\
& \left.=e_{2} e_{1}=e_{2} e_{2}=0\right\rangle .
\end{aligned}
$$

ii) $A$ 是不可换的:

$$
\text { (NI) } A=\left\langle e_{1}, e_{2} ! e_{1} e_{1}=e_{1} e_{2}=0, e_{2} e_{1}\right.
$$$$
\left.=-e_{1}, e_{2} e_{2}=-e_{2}\right\rangle \text {; }
$$

$$
\begin{aligned}
\text { (NII)A } A & \in\left\{A_{k} \mid A_{k}=\left\langle e_{1}, e_{2}\right| e_{1} e_{1}=e_{1} e_{2}\right. \\
& \left.\left.=0, e_{2} e_{1}=-e_{1}, e_{2} e_{2}=k e_{2}\right\rangle, k \neq-1\right\} \\
A_{-1} & =\left\langle e_{1}, e_{2}\right| e_{1} e_{1}=e_{1} e_{2}=0, e_{2} e_{1} \\
& \left.=-e_{1}, e_{2} e_{2}=e_{1}-e_{2}\right\rangle ; \\
(N \mathrm{III}) A & =\left\langle e_{1}, e_{2}\right| e_{1} e_{1}=0, e_{1} e_{2}=e_{1}, e_{2} e_{1} \\
& \left.=0, e_{2} e_{2}=e_{2}\right\rangle ; \\
(\mathrm{NIV}) A & \in\left\{A_{l} \mid A_{l}=\left\langle e_{1}, e_{2}\right| e_{1} e_{1}=0, e_{1} e_{2}\right. \\
& =l e_{1}, e_{2} e_{1}=(l-1) e_{1}, e_{2} e_{2} \\
& \left.\left.=e_{1}+l e_{2}\right\rangle, l \neq 0\right\} ; \\
(N V) A & =\left\langle e_{1}, e_{2}\right| e_{1} e_{1}=2 e_{1}, e_{1} e_{2}=e_{2}, e_{2} e_{1} \\
& \left.=0, e_{2} e_{2}=e_{1}\right\rangle .
\end{aligned}
$$

其中 $N$ I, $N$ III 型是结合代数, $N$ II,$N$ IV, $N V$ 型是非结合的左对称代数.

推论 1 若维左对称代数 $A$ 不存在非 平凡理想, 则 $A$ 一定同构于定理 1 中的 $N V$ 型左对称代数.

\section{白承铭孟道貲}

(南开大学数学系, 天津 300071)

\section{氧化物载体表面结构对离子化合物分散的影响}

负载型催化剂因其广泛的工业应用而受 到研究重视. Knozinger ${ }^{[1]}$, Hercules ${ }^{[2]}$ 以及
唐有祺 ${ }^{[3]}$ 等都对负载型氧化物表面组分分 散后的存在状态及分散的实质进行了研究, 
认为活性组分分散后在载体表面形成单层、 亚单层或多层. 形成分散态的原因是由于固 -固润湿所引起的表面能减少或形成表面阴 离子密置单层结构. 我们曾对一些离子化合 物在 $\gamma-\mathrm{Al}_{2} \mathrm{O}_{3}$ 和 $\mathrm{CeO}_{2}$ 载体表面的分散进行 过讨论 ${ }^{[4,5]}$, 认为焙烧过程促使这类化合物 在载体表面扩散, 而形成分散态表面物种的 实质是金属阳离子进入载体表面空位, 与之 相伴的阴离子覆盖其上以保持电中性.

最近, 我们选用一系列具有不同表面结 构的氧化物载体 $\gamma-\mathrm{Al}_{2} \mathrm{O}_{3}, \mathrm{CeO}_{2}, \mathrm{TiO}_{2}$ (锐 钛), $\mathrm{MgO}$ 和 $\mathrm{SiO}_{2}$ 经 $600{ }^{\circ} \mathrm{C}$ 预处理后, 与化合 物 $\mathrm{NaBr}$ 分别按 $1.0 \mathrm{mmol} \mathrm{NaBr} / 100 \mathrm{~m}^{2}$ (载 体)的比例充分混合, 并在空气气氛下于 $500{ }^{\circ} \mathrm{C}$ 焙烧 $24 \mathrm{~h}$, 采用 XRD 考察了 $\mathrm{NaBr}$ 在 这些载体上的分散行为, 研究结果表明, $\mathrm{NaBr}$ 的晶相衍射峰在焙烧后的样品 $\mathrm{NaBr} /$ $\gamma-\mathrm{Al}_{2} \mathrm{O}_{3}, \mathrm{NaBr} / \mathrm{CeO} 2, \mathrm{NaBr} / \mathrm{TiO}_{2}$ 中完全消 失, 说明在这些具有表面空位的载体上可以 形成表面分散态的 $\mathrm{NaBr}$, 分散后的 $\mathrm{NaBr}$ 可 用图来表示(图略). 而在没有表面空位的载
体 $\mathrm{MgO}$ 和 $\mathrm{SiO}_{2}$ 表面 $\mathrm{NaBr}$ 不能分散, 即焙烧 前后 $\mathrm{NaBr}$ 的晶相衍射峰不变. 这些事实进 一步说明了离子化合物在载体表面分散的实 质就是金属阳离子进入载体表面空位的观 点.

\section{参考文 献}

1 Knozinger H, Taglauer E. Catalysis, Vol 10. London: The Royal Society of Chemistry, 1993. 1 40

2 Burggaf L W, Leyden D E, Hercules D M et al. Photoacoutic spectroscopy study of metal-support interactions in $\mathrm{Co} / \gamma-\mathrm{Al}_{2} \mathrm{O}_{3}$ catalysts. J Catal, 1982, 78: 360 379

3 Xie Y C, Tang Y Q. Spontaneous monolayer dispersion of oxides and salts onto surfaces of supports, applications to heterogeneous catalisis. Adv Catal, 1990, 37: 1 43

4 Chen Y, Zhang L F. Surface interaction model of $\gamma$-alumina-supported metal oxides. Catal Lett, 1992, 12: 51 62

5 董 林, 陈 政. 一些离子化合物在 $\mathrm{CeO}_{2}$ 和 $\gamma-\mathrm{Al}_{2} \mathrm{O}_{3}$ 载体上的分散-咲入模型的新证据. 催化学报, 1995 , 16: $85 \sim 86$

\section{董林徐斌胡静秋陈 懿} (南京大学化学系, 南京 210093) 